BUHEP-92-25

HUTP-92/A033

hep-ph/9207249

\title{
Electroweak Corrections in Technicolor Reconsidered
}

\author{
R. Sekhar Chivukula ${ }^{a, 1}$ \\ Michael J. Dugan ${ }^{a, b, 2}$ \\ and
}

Mitchell Golden ${ }^{a, 3, *}$

\begin{abstract}
Radiative corrections to electroweak parameters in technicolor theories may be evaluated by one of two techniques: either one estimates spectral function integrals using scaled QCD data, or one uses naive dimensional analysis with a chiral Lagrangian. The former yields corrections to electroweak parameters proportional to the number of flavors and the number of colors, while the latter is proportional to the number of flavors squared and is independent of the number of colors. We attempt to resolve this apparent contradiction by showing that the spectrum of technicolor one obtains by scaling QCD data to high energies is unlikely to resemble that of an actual technicolor theory. The resonances are likely to be much lighter than naively supposed and the radiative corrections to electroweak parameters may by much larger. We also argue that much less is known about the spectrum and the radiative corrections in technicolor than was previously believed.
\end{abstract}

$7 / 92$

\footnotetext{
${ }^{a}$ Boston University, Department of Physics, 590 Commonwealth Avenue, Boston, MA 02215

${ }^{b}$ Lyman Laboratory of Physics, Harvard University, Cambridge, MA 02138

${ }^{1}$ sekhar@weyl.bu.edu, ${ }^{2}$ dugan@huhepl.bitnet, ${ }^{3}$ golden@weyl.bu.edu

*Address after Sept 1, 1992: Lyman Laboratory of Physics, Harvard University, Cambridge,
} MA 02138 


\section{Introduction}

Because of the increased precision with which quantities like the $W$ and $Z$ masses are known, there has recently been a great deal of interest in constraining new physics by its radiative effects. The simplest kinds of radiative corrections are "oblique" [1], meaning that they affect only the propagator of the electroweak gauge bosons. If the new physics is heavy enough, then the radiative effects are quantified in three parameters [2], commonly referred to as $S, T$, and $U$. Much work has focused on the evaluation of these quantities in technicolor theories [3].

Since technicolor is a strongly interacting theory, the corrections cannot be evaluated by ordinary perturbation theory. One of two approaches is usually followed. The first involves expressing $S$, for example, as a spectral integral, which is then evaluated by taking data from QCD experiments and scaling it to technicolor energies [2] [4] [5]. The second is to use chiral Lagrangian techniques [6]. In the latter case $S, T$, and $U$ are related to coefficients of four-derivative operators in a chiral Lagrangian [7] [8] [9] [10] [11] [12]. In general, the coefficients of a chiral Lagrangian are arbitrary, but they may be estimated by the technique of naive dimensional analysis (NDA) [13] which states that, roughly speaking, the size of a four-derivative term in the chiral Lagrangian is set by the typical size of the PGB loops that contribute to it.

When applied to the one-family model of technicolor [14], both these techniques yield contributions to the radiative parameters of about the same size. Yet there is something a bit odd about this concurrence of the results. The two techniques appear to depend differently on the numbers of colors and families involved. As we will explain below, the spectral function technique, as normally implemented, yields values of $S$ proportionalt to $N_{D} N_{T C}$, where $N_{D}$ is the number of doublets (four in the one-family model), and $N_{T C}$ is the number of technicolors in the underlying gauge theory. The chiral Lagrangian with NDA, on the other hand, certainly knows nothing about the number of colors in the underlying theory. We will show below that the corrections grow like $N N_{D}$, where $N$ is the total number of technifermion flavors.

This note is an attempt to reconcile these two picturesl. We will argue that the scaling of QCD data which one does in deriving $S$ is based on an unwarranted assumption

1 A third approach to evaluating $S$ in technicolor theories is based on a direct estimate of the technihadronic contribution to the $W$ and $Z$ vacuum polarization diagrams [15] [16]. This approach also produces a value of $S$ proportional to $N_{D} N_{T C}$.

2 Ref [5] also addresses this issue. However, their point of view is rather different from ours. 
about the spectrum of resonance masses in technicolor theories. In particular, we argue that naively scaling the masses of the resonances in QCD will underestimate the masses of mesons in technicolor. If this is so, the radiative corrections may be considerably larger than given by the spectral function estimajtes. We will argue that the $N N_{D}$ dependence is probably applicable when $N_{T C}$ is small, while $N_{D} N_{T C}$ holds only for rather large values of $N_{T C}$.

In any case, we argue that much less is known about the spectrum of technicolor than was previously believed. Though the most pessimistic evaluations of the viability of the simplest technicolor models may in the end be justified, more uncertainty remains in the evaluation of the radiative corrections than was appreciated.

The plan of this paper is as follows. In the next section we explain in detail how the factors of $N_{D}$ and $N_{T C}$ come about in the different techniques. The following section we explain an idea suggested by Kaplan [17] which points the way to resolving the conflict. The following section applies the argument to technicolor theories. In section five we apply these considerations in the limit of QCD in which both $N$ and $N_{T C}$ go to infinity simultaneously, and make some concluding remarks in section six.

\section{The Two Calculations}

The quantity $S$ is defined by 3

$$
S=-\left.16 \pi \frac{\partial}{\partial q^{2}} \Pi_{3 Y}\left(q^{2}\right)\right|_{0},
$$

where $\Pi_{3 Y}$ is the transverse part of the weak- $T_{3}$-current-weak-hypercharge-current twopoint correlator. In this paper we will consider only the case of technicolor theories in which the symmetry breaking pattern is $S U(N)_{L} \times S U(N)_{R} \times U(1) \rightarrow S U(N)_{V} \times U(1)$. We embed the weak $S U(2)_{W}$ into $S U(N)_{L}$ as $N_{D}$ doublets and $N_{S}=N-2 N_{D}$ singlets. This embedding preserves a custodial $S U(2)_{C}$ symmetry. In this case, we may rewrite $S$ as

$$
S=-\left.4 \pi \frac{\partial}{\partial q^{2}}\left(\Pi_{V V}\left(q^{2}\right)-\Pi_{A A}\left(q^{2}\right)\right)\right|_{0},
$$

where $\Pi_{V V}\left(\Pi_{A A}\right)$ is the transverse part of the two-point correlator of the third component of the weak vector (axial) current. Defined this way $S$ is infinite; however by subtracting

3 This discussion of the spectral integral computation follows ref [5]. 
the same expression in the standard one-Higgs model (with some fixed value of the Higgs boson mass) one may define a finite quantity which parameterizes the radiative effects.

The Fermi constant is given by

$$
\frac{G_{F}}{\sqrt{2}}=\frac{1}{2 \Pi_{A A}(0)}
$$

so we identify $\Pi_{A A}(0)=v^{2}=(246 \mathrm{GeV})^{2}$. Because of the embedding of the weak gauge currents, we see $v^{2}=N_{D} f^{2}$, where $f$ is the technipion decay constant.

One may rewrite $S$ as an integral over a spectral function

$$
S=\frac{1}{3 \pi} \int_{0}^{\infty} \frac{d s}{s}\left\{\left[R_{V}(s)-R_{A}(s)\right]-\frac{1}{4}\left[1-\left(1-m_{H}^{2}\right)^{3} \theta\left(s-m_{H}^{2}\right)\right]\right\},
$$

where $m_{H}$ is the reference value of the Higgs boson mass and

$$
\begin{aligned}
& R_{V}(s)=-12 \pi \operatorname{Im} \frac{\Pi_{V V}(s)}{s} \\
& R_{A}(s)=-12 \pi \operatorname{Im} \frac{\Pi_{A A}(s)-\Pi_{A A}(0)}{s} .
\end{aligned}
$$

The second term in (2.4) subtracts off the standard model contribution and renders the expression finite.

The integral in (2.4) is very convergent in the ultraviolet, and therefore it is most sensitive to the behavior of the $R$ 's in the infrared. A simple model for the functions $R_{V}$ and $R_{A}$ is that they are each concentrated at their lightest resonance 4 :

$$
\begin{aligned}
& R_{V}(s)=12 \pi^{2} F_{\rho T} \delta\left(s-m_{\rho T}^{2}\right) \\
& R_{A}(s)=12 \pi^{2} F_{a_{1} T} \delta\left(s-m_{a_{1} T}^{2}\right),
\end{aligned}
$$

where the $F$ s have dimensions of mass, and $m_{\rho T}$ and $m_{a_{1} T}$ are the techni- $\rho$ and techni$a_{1}$ masses respectively. The first and second Weinberg sum rules [18] imply relationships among these quantities [19]

$$
\begin{array}{r}
F_{\rho T}=\frac{m_{a_{1} T}^{2} N_{D} f^{2}}{m_{a_{1} T}^{2}-m_{\rho T}^{2}} \\
F_{a_{1} T}=\frac{m_{\rho T}^{2} N_{D} f^{2}}{m_{a_{1} T}^{2}-m_{\rho T}^{2}} .
\end{array}
$$

4 This model has no infrared problem, so we neglect the weak dependence on $m_{H}$ in the following discussion. 
One therefore obtains

$$
S=4 \pi\left(1+\frac{m_{\rho T}^{2}}{m_{a_{1} T}^{2}}\right) \frac{N_{D} f^{2}}{m_{\rho T}^{2}}
$$

In going from QCD to technicolor holding $N_{T C}$ fixed at 3, one scales the masses of the vector technimesons so that $f / m$ is fixed [20], and so $S$ is proportional to one power of $N_{D}$. To go to some other value of $N_{T C}$ one uses the large- $N_{c}$ QCD [21] result that the ratio $f / m$ scales like $\sqrt{N_{T C}}$. Plugging in the observed masses of the $\rho$ and $a_{1}$, one obtains the result

$$
S \sim 0.083 N_{D} N_{T C}
$$

A more elaborate analysis which includes the width of the vector technimesons gives roughly the same answer and has the same dependence on $N_{D}$ and $N_{T C}$.

Next we turn to the computation in the chiral Lagrangian. The two-point function $\Pi_{3 Y}$ has two contributions. At tree level, the relevant number is the coefficient司 $c$ of the operator $W_{3}^{\mu \nu} B_{\mu \nu}$, where $W_{i}^{\mu \nu}$ and $B^{\mu \nu}$ are the $S U(2)_{W}$ and $U(1)_{Y}$ field strengths respectively. The definition is

$$
S=-32 \pi c .
$$

There are additional, formally infinite, contributions to $\Pi_{3 Y}$ arising from loops of technipions. These divergences may be absorbed into a renormalization of $c$ in the usual way, and it is the sum of the loop contributions to $\Pi_{3 Y}$ and that from $c$ that is finite. Because of the three massless exact Goldstone bosons which are "eaten" by the $W$ and $Z$, there is an infrared logarithmic divergence. As above however, the same divergence exists in the standard model, and the same subtraction renders $S$ finite.

Using naive dimensional analysis we bound $S$ as follows. One calculates the graphs consisting of a loop of technipions, which induce a running of $c$. The technipions contribute to the running from a high scale $\Lambda_{\chi}$, at which the chiral Lagrangian breaks down, down to their mass, at which point they are integrated out of the theory. Without a fine tuning, it is inconsistent to assume that $S$ is smaller than this logarithmic contribution.

The technipions form (approximately) degenerate multiplets under the custodial $S U(2)_{C}$; each one makes a contribution to $S$ of [9]

$$
\Delta S=\frac{1}{36 \pi} \ell(\ell+1)(2 \ell+1) F \log \left(\frac{\Lambda_{\chi}^{2}}{m_{\pi}^{2}}\right),
$$

5 This is related to the coefficient called $\ell_{10}$ in the notation of Gasser and Leutwyler [22]. They also point out that in the large- $N_{c}$ limit, $\ell_{10}$ is proportional to $N_{c}$. 
where $\ell$ is the isospin of the multiplet under the $S U(2)_{c}$, and $F$ is a symmetry factor which is 1 for a non-self conjugate multiplet (like the $K^{+}, K^{0}$ of QCD), and $1 / 2$ for a self-conjugate multiplet (like $\pi^{+}, \pi^{0}, \pi^{-}$). The models we are considering have $N_{D}^{2}-1$ self-conjugate triplets $(\ell=1)$ of massive technipions, $N_{D} N_{S}$ non-self-conjugate doublets $(\ell=1 / 2)$, $N_{D}^{2}+N_{S}^{2}-1$ singlets $(\ell=0)$, and one triplet of "eaten" technipions. The contribution of this last set is partially cancelled by the standard model subtraction, leaving a term proportional to $\log \left(\Lambda_{\chi}^{2} / m_{H}^{2}\right)$, which we neglect. Therefore we may write

$$
S \geq \frac{1}{24 \pi}\left(N N_{D}-2\right) \log \left(\frac{\Lambda_{\chi}^{2}}{m_{\pi}^{2}}\right)
$$

At this stage the large scale $\Lambda_{\chi}$ is arbitrary, but in an $S U(N)_{L} \times S U(N)_{R} \times U(1) \rightarrow$ $S U(N)_{V} \times U(1)$ chiral lagrangian it cannot be larger 6 than of order $4 \pi f / \sqrt{N}$ [23]. Consider the case of the one-family model $\left(N=8, N_{D}=4\right)$. If we assume that this bound is saturated then $\Lambda_{\chi}$ is about $550 \mathrm{GeV}$. If we take all the technipions to have a mass of about $100 \mathrm{GeV}$, then $S$ is bigger than or about 1, just as it was when it was evaluated using the spectral integrals in the vector dominance model.

As was stressed in the introduction, this numerical coincidence is rather surprising. The chiral Lagrangian calculation displays no dependence on $N_{T C}$, but goes as $N_{D} N-2$ (ignoring the weak dependence in the logarithm). The vector dominance model computation went like $N_{D} N_{T C}$. Certainly the two computations will be very different if we go to the two-family model, for example, or even add some extra singlets.

We may explain the discrepancy by considering the graphs which the two calculations have included. The vector dominance model scaled with large- $N_{C}$ QCD includes the class

of diagrams denoted in fig. 1, which goes as $N N_{T C}$. The class of diagrams included in the chiral Lagrangian is shown in fig. 2 - proportional to $N N_{D}$. Were we able to compute $S$ in the full TC theory, both classes would be present, the outstanding question is which one dominates.

\section{Kaplan's Argument}

Imagine that it is possible to solve exactly an $S U\left(N_{T C}\right)$ gauge theory with $N$ flavors of fermions. We assume that the fermions are confined, and that the observed spectrum

${ }^{6}$ In ref [9], the $\Lambda_{\chi}$ was taken to be $4 \pi f$. This is too large a scale. 
consists entirely of TC singlets. The $S U(N)_{L} \times S U(N)_{R} \times U(1)$ chiral symmetry breaks to $S U(N)_{V} \times U(1)$ through the formation of a condensate. Aside from the $N^{2}-1$ technipions, all the other particles are massive. Make a plot of the mass $M$ of the lightest massive resonance divided by the pion decay constant. As $N_{T C}$ goes to infinity with $N$ fixed, we know that this ratio goes as $1 / \sqrt{N_{T C}}$ [21]. So if we know $M$ for a theory in which $N_{T C}$ is large, as we reduce $N_{T C}$ the value of $M / f$ increases.

On the other hand, as argued in [24], there is an upper bound to $M / f$. In a lowestorder chiral Lagrangian computation of the $\pi \pi \rightarrow \pi \pi$ scattering process, the amplitude for the $S U(N)_{V}$ singlet spin-0 partial wave is given by [25]

$$
a=\frac{N s}{32 \pi f^{2}}
$$

where $s$ is the usual Mandelstam variable. A partial wave amplitude must lie on or inside the Argand circle, so when $s$ is greater than or about $4 \pi f / \sqrt{N}$, the corrections must be bigger than the lowest order computation, indicating the likely divergence of the chiral Lagrangian's expansion of amplitudes as a power series in energy. This is the probable scale for the formation of non-analytic structure in the $S$-matrix, such as resonances. It is unlikely that it is possible to postpone such structures much beyond this mass, though they may be lighter.

$$
\frac{M}{f} \leq \frac{4 \pi}{\sqrt{N}}
$$

Once the mass of the lightest resonance saturates this bound, decreasing $N_{T C}$ cannot increase $M / f$.

A plot of $M / f$ as a function of $N_{T C}$ might look something like one of the lines in

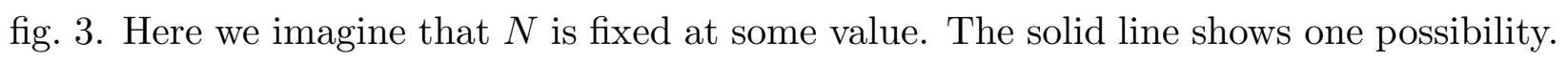
At large $N_{T C}$, the curve goes as $1 / \sqrt{N_{T C}}$. At somewhat lower $N_{T C}$, however, there is a flat part where the mass of the lightest resonance saturates the bound (3.2) and changing $N_{T C}$ cannot much affect $M / f$. At very low $N_{T C}$, the theory loses asymptotic freedom and ceases to make sense. Another possibility is shown as the dashed line. Here $M / f$ is always on the falling part of the curve, and the bound (3.2) is never saturated.

In QCD as we know it, with two light flavors 1 and $N_{c}=3$, the scale $4 \pi f / \sqrt{N}$ is about $825 \mathrm{MeV}$. The lightest resonances, such as the $\rho(770)$, have masses of about this value and

7 One may wish to argue that there are three light quarks in QCD. Since the bound is only a rough guide, it does not distinguish between $N=2$ and $N=3$. 
appear therefore to be saturating the bound. Therefore, in QCD as we know it, we are on a curve like the solid one, and $N_{c}=3$ corresponds to a point like $A$ or $B$, not $C$ ! If QCD is at a point like $\mathrm{A}$, one has to increase $N_{c}$ well beyond 3 before the $1 / \sqrt{N_{c}}$ dependence sets in.

\section{Application to Electroweak Corrections}

In the one-family model $4 \pi f / \sqrt{N}$ is about $550 \mathrm{GeV}$, and we expect resonances of this mass or lower. On the other hand, the vector dominance computation assumed that the technicolor spectrum was directly analogous to the QCD spectrum and that (for $N_{T C}=3$ ) the ratio $m_{\rho T} / f$ was the same as in QCD, yielding $m_{\rho T}=1 \mathrm{TeV}$. Such a large mass for the lightest resonance is inconsistent. The lightest resonance (which may or may not be a vector meson) must be lighter than the simple scaling suggests.

Suppose that we continue to make the (entirely unwarranted) assumption that the spectrum of technicolor looks just like that of QCD, i.e. we model the $V V$ and $A A$ spectral functions at small $q^{2}$ as each being dominated by a single spin-1 resonance. However, instead of scaling $M / f$ from QCD, assume that we are at a point like A or B in fig. 3 , and put $m_{\rho T} \approx 4 \pi f / \sqrt{N}$. Evaluating eqn. (2.8) we find:

$$
S=\frac{1}{4 \pi}\left(1+\frac{m_{\rho T}^{2}}{m_{a_{1} T}^{2}}\right) N N_{D}
$$

The dependence of $S$ has lost one factor of $N_{T C}$, and gained a factor of $N$. In other words, the dependence on $N, N_{D}$, and $N_{T C}$ is the same as in the chiral Lagrangian calculation using NDA!

Another possibility is that $N_{T C}$ is sufficiently large that we are at a point like C, or that technicolor is on the dashed curve. In this case, the vector dominance assumption gives a dependence like $N_{D} N_{T C}$. However, if the techni- $\rho$ is the lightest resonance, its mass is less than $550 \mathrm{GeV}$, and the scaling of $M / f$ from QCD is invalid.

\section{Double Scaling Limit}

In QCD neither $N$ nor $N_{c}$ is particularly large, and it is not clear that the large $N_{c}$ approximation is particularly good. Moreover, the ratio $N / N_{c}$ is not small, and it may not be possible to neglect it. In one family technicolor this problem is exacerbated, since there are eight light fermions, rather than two. 
Consider instead the limit of QCD in which $N_{c}$ and $N$ are both taken to infinity with their ratio held fixed [26]. The graphs that contribute now are planar, but with holes for fermion loops. The double scaling limit is a better approximation to QCD than the ordinary large- $N_{c}$ QCD limit, in the sense that all the diagrams leading in $N_{c}$ are included, plus some extra ones. If one believes that QCD is well approximated by its large- $N_{c}$ limit, then the double scaling limit is also justified.

In this double limit, fig. 1 and fig. 2 are both the same order, so it is not possible to know which one is larger. Both (2.9) and (2.12) have the same dependence. In the double scaling limit, the ratios of the masses of the resonances to $f$ falls like $1 / \sqrt{N_{T C}}$ as before, but the ratio of the masses to the widths stays fixed. This is because the number of open channels into which the resonances decay grows with $N$.

If we believe that QCD with $N_{c}=3$ and $N=2$ is close to the double scaling limit, then the one-family model most closely resembles QCD at $N_{T C}=12$. Therefore (in the double scaling limit) when $N_{T C}=12$, the one family model is at A or B, not C. Since QCD saturated the bound on $M / f$, all one-family models with $N_{T C} \leq 12$ must also saturate the bound.

For the one-family model with $N_{T C}=12$, (4.1) yield $8 \Omega \approx 4$. If vector dominance continues to work when $N_{T C}$ is less than 12 , and if the lightest resonance is still the techni$\rho$, and if it continues to saturate the bound on its mass, then reducing $N_{T C}$ will not reduce the value of $S$. The electroweak corrections in one-family technicolor could be far larger than was previously estimated.

On the other hand a great deal of caution should be advised. The use of vector dominance in a model with a radically different, larger, value of $N / N_{T C}$ is highly speculative. Kaplan's argument discusses only the scale at which the lightest resonance forms - not the quantum numbers of the lightest resonance or the behavior of the theory at higher energies. There is no reason to believe (as (4.1) seems to imply) that all $S U\left(N_{T C}\right)$ gauge theories with $N_{T C}$ in the flat part of the curve are identical, or even similar. For example, as suggested by Cahn and Suzuki, the lightest resonance could be a scalar instead of a vector particle [25] [27]. It is possible that the scalar's appearance delays the formation of the techni- $\rho$ to a somewhat higher scale. We really do not know very much about the spectrum of technicolor theories.

8 Recall that NDA gives a lower bound on $S$, and this estimate is consistent with it. 
Lastly, the masses of the technipions may be very different from the scaled value of the pion mass. Certainly this will affect the size of the chiral logs. More importantly, if the technipions are sufficiently heavy, they decouple from the low-energy amplitudes, effectively reducing the number of flavors and increasing the bound on the mass of the lightest resonance. This is why the $\rho$ has a mass of $770 \mathrm{MeV}$, well above the $475 \mathrm{MeV}$ bound on its mass it would have if all six quarks were light. It may be possible to construct a technicolor model which avoids large radiative corrections if the technipions are heavy enough.

\section{Conclusions}

Kaplan's argument addresses a longstanding question: How are NDA and the large$N_{c}$ expansion consistent? The diagrams used in NDA are always subleading in a large- $N_{c}$ expansion, and yet dimensional analysis generally gives reasonable values for the sizes of coefficients of the higher dimension operators in the chiral Lagrangian of QCD. The arguments given here show that NDA will work to estimate the sizes of these coefficients for values of $N_{c}$ such that the theory is on the flat part of the curve in fig. 3, at a point like A or B. It appears that ordinary QCD with $N_{c}=3$ is such a theory.

We have seen that there is a simple way to reconcile the vector dominance and chiral Lagrangian/NDA calculations of $S$. If, as we have argued, technicolor and QCD are on the flat part of the curve in fig. 3 , then the dependence on $N$ and $N_{D}$ suggested by the chiral Lagrangian is appropriate. If, on the other hand, a technicolor model has values of $N_{T C}$ which makes the masses of the mesons much lighter than $4 \pi f / \sqrt{N}$, then that theory is on the falling part of the curve, and scaling the radiative corrections with $N_{T C}$ may be justified. In either case, taking $M / f$ from QCD is invalid.

At first glance, it seems that the radiative corrections in one-family technicolor may be much larger than previously estimated. On the other hand, we prefer to advise caution, since technicolor is not just QCD scaled up. It is a much less familiar theory than was assumed.

\section{Acknowledgements.}

We thank Howard Georgi, David Kaplan, Aneesh Manohar, and Michael Peskin for useful conversations. R.S.C. acknowledges the support of a NSF Presidential Young Investigator Award, an Alfred P. Sloan Foundation Fellowship, and a DOE Outstanding 
Junior Investigator Award and thanks the Aspen Center for Physics for its hospitality during the completion of this work. M.G. and R.S.C. thank the Texas National Research Laboratory Commission for support under Superconducting Super Collider National Fellowships. This research is supported in part by the National Science Foundation under grants PHY-87-14654 and PHY-90-57173, by the Texas National Research Laboratory Commission, under grants RGFY9206 and RGFY92B6, and by the Department of Energy, under contract numbers DE-AC02-89ER40509 and DE-FG02-91ER40676. 


\section{References}

[1] B. Lynn, M. Peskin, and R. Stuart, in Trieste Electroweak 1985 Proceedings.

[2] M. Peskin and T. Takeuchi, Phys. Rev. Lett. 65 (1990) 964.

[3] S. Weinberg, Phys. Rev. D 19 (1979) 1277;

L. Susskind, Phys. Rev. D 20 (1979) 2619.

[4] R. N. Cahn and M. Suzuki, Phys. Rev. D 44 (1991) 3641.

[5] M. Peskin and T. Takeuchi, Phys. Rev. D 46 (1992) 381.

[6] S. Weinberg, Phys. Rev. 166 (1968) 1568;

S. Coleman, J. Wess, and B. Zumino, Phys. Rev. 177 (1969) 2239 ;

C. Callan, S. Coleman, J. Wess, and B. Zumino Phys. Rev. 177 (1969) 2246.

[7] A. Longhitano, Phys. Rev. D 22 (1980) 1166 and Nucl. Phys. B188 (1981) 118.

[8] R. Renken and M. Peskin, Nucl. Phys. B211 (1983) 93.

[9] M. Golden and L. Randall, Nucl. Phys. B361 (1990) 3.

[10] B. Holdom and J. Terning, Phys. Lett. B247 (1990) 88.

[11] A. Dobado, D. Espriu, and M. J. Herrero, Phys. Lett. B255 (1990) 405.

[12] H. Georgi, Nucl. Phys. B363 (1991) 301.

[13] S. Weinberg, Physica 96A (1979) 327;

see also H. Georgi and A. Manohar, Nucl. Phys. B234 (1984) 189 and H. Georgi and L. Randall, Nucl. Phys. B276 (1986) 241.

[14] E. Farhi and L. Susskind, Phys. Rev. D 20 (1979) 3404.

[15] T. Appelquist and G. Triantaphyllou, Phys. Lett. B278 (1992) 345.

[16] R. Sundrum and S. Hsu, "Walking Technicolor and Electroweak Radiative Corrections", LBL preprint LBL-31066, UCB-PTH-91/34 (June 1992).

[17] D. Kaplan, private communication

[18] C. Bernard et al., Phys. Rev. D 12 (1976) 792.

[19] S. Weinberg, Phys. Rev. Lett. 18 (1967) 507.

[20] S. Dimopoulos, S. Raby, and G. Kane, Nucl. Phys. B182 (1981) 77

[21] G. 't Hooft, Nucl. Phys. B72 (1974) 461.

[22] Gasser and Leutwyler, Ann. Phys. (NY) 158 (1984) 142 and Nucl. Phys. B250 (1985) 465.

[23] M. Soldate and R. Sundrum, Nucl. Phys. B340 (1990) 1.

[24] R. S. Chivukula, M. Dugan, and M. Golden, "Analyticity, Crossing Symmetry, and the Limits of Chiral Perturbation Theory", Boston University preprint BUHEP-92-18 (June 1992).

[25] R. N. Cahn and M. Suzuki, Phys. Rev. Lett. 67 (1991) 169.

[26] G. Veneziano, Nucl. Phys. B117 (1976) 519;

G. Veneziano, in Proceedings of the Twelfth Rencontre de Moriond, Flaine-hauteSavoie (France), Vol. III, Tran Thanh Van, ed., 1977, p 113-134

[27] R. S. Chivukula and M. Golden, Nucl. Phys. B372 (1992) 44. 


\section{Figure Captions}

Fig. 1. The class of diagrams that is included by the large- $N_{c}$ vector dominance approximation. Gluon lines are omitted.

Fig. 2. The class of diagrams that is included in the chiral Lagrangian computation. Gluon lines are omitted.

Fig. 3. Hypothetical graphs of the mass of the lightest resonance divided by the pion decay constant in an $S U\left(N_{T C}\right)$ gauge theory with a fixed number $N$ of fermions as a function of $N_{T C}$. 


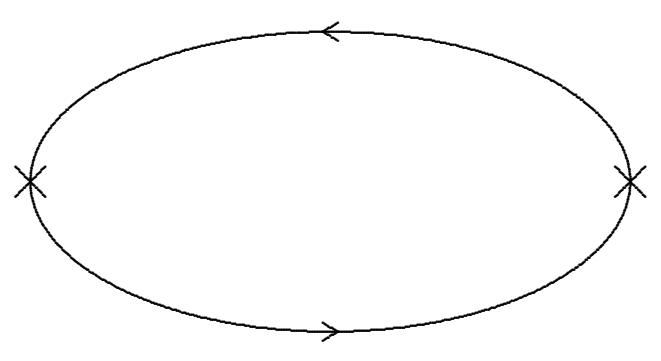




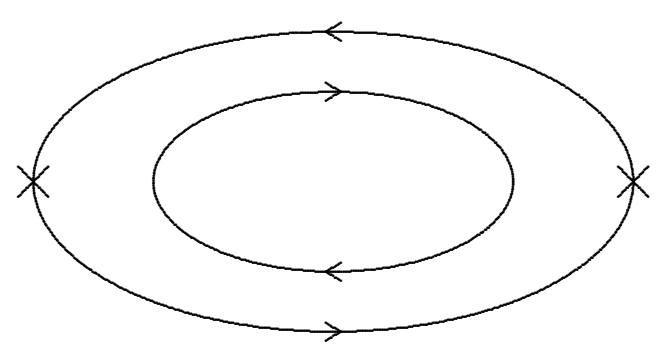




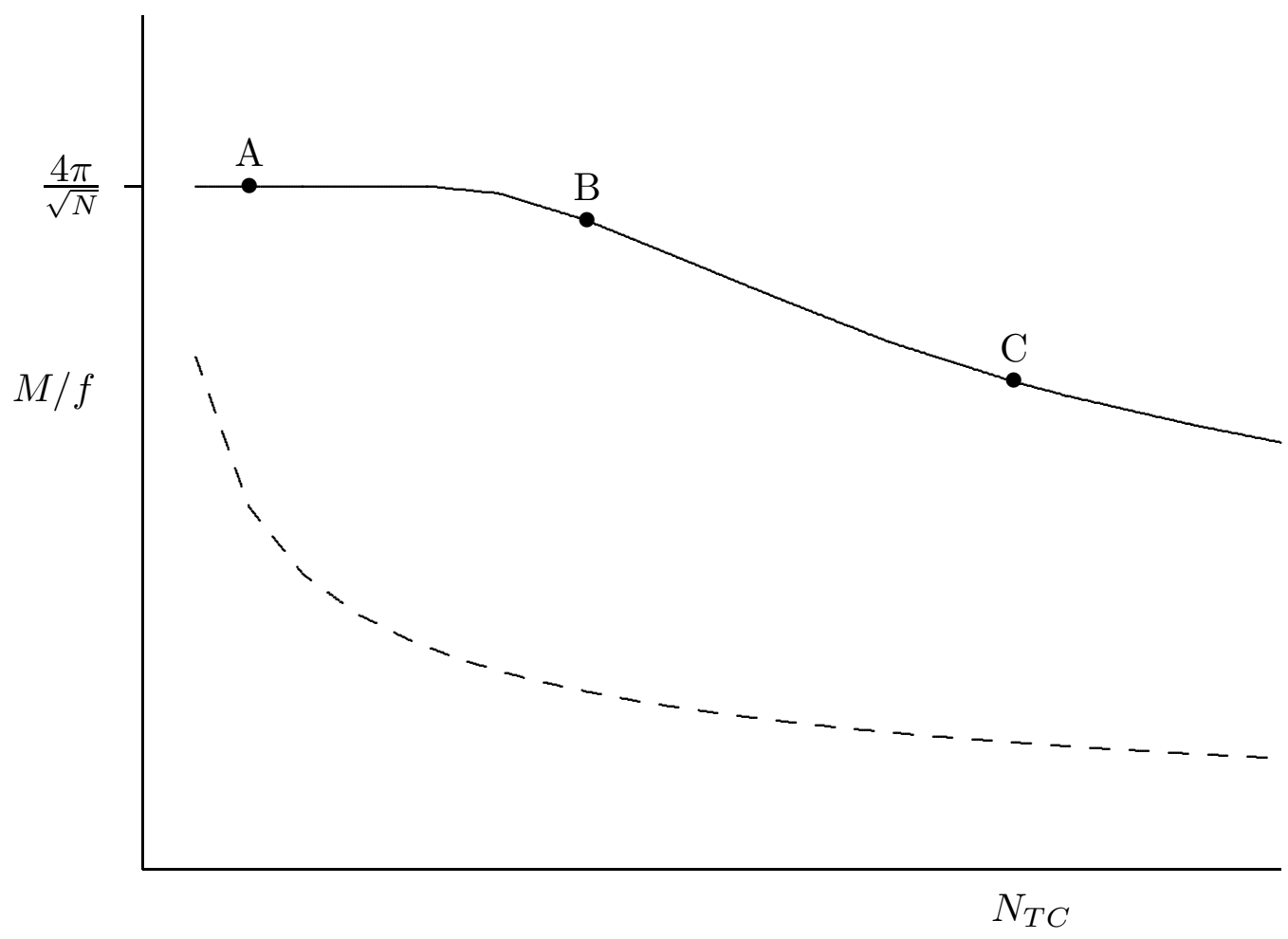

